\title{
POTENTIAL-BASED BOUNDARY ELEMENT METHOD TO \\ CALCULATE THE HYDRODYNAMIC DRIFT FORCE ON THE FLOATING CYLINDER
}

\author{
Hamidreza Ghafari ${ }^{1}$, Mohammad Motallebi ${ }^{2}$, Hassan Ghassemi ${ }^{3}$ \\ ${ }^{1,3}$ Department of Maritime Engineering, Amirkabir University of Technology, Tehran, Iran \\ ${ }^{2}$ Department of Civil and Environmental Engineering, Amirkabir University of Technology \\ Tehran, Iran \\ hamidrezaghafari@aut.ac.ir,m_motallebi@aut.ac.ir,gasemi@aut.ac.ir
}

Received: 7 December 2019; Accepted: 4 October 2020

\begin{abstract}
This paper presents the calculation of the hydrodynamic drift force by using the potetial-based boundary element method (BEM). The potential theory and far-field wave drift forces solution will be described. The comparison of non-dimensional drift force for surge and heave motions are in good agreement between numerical and experimental data. The effect of different drafts and the radius of a cylinder on the drift forces (surge, heave and pitch) are presented and discussed.
\end{abstract}

MSC 2010: 31B25, 76B15, 76B07

Keywords: floating cylinder, drift forces, boundary element method

\section{Introduction}

Second-order and low-frequency wave forces have an important influence on the dynamics of floating objects. These forces are known as wave drift forces and the results in the steady slow drift motion along the wave direction. Although the first-order wave forces involve a significant portion of body excitation, the second-order theories are important in some surrounding circumstances to calculate the nonlinear effects of the wave. In these theories, the condition of flow impermeability into the object on the wetted surface is considered more precise. In the solving procedure of second-order equations, all terms related to the velocity potential function, fluid pressure and wave forces which have a linear relationship with both wave amplitude and second power of wave amplitude are considered. Furthermore, the nonlinear velocity effects of fluid particles on a free surface will also be considered. Second-order equations include moderate forces and oscillating forces with frequencies greater or less than the frequency of the wave.

In general, research on the second-order wave forces can be divided into four categories [1]. The first set of methods based on the potential theory is considering 
the law of momentum conservation and energy in the surrounding fluid of a float object. In these methods, changes in fluid momentum with the average fluid force on a float object are considered equal, which are called far-field methods. The second group of methods is based on the potential theory method, which, by direct integration, calculates the fluid pressure on the floating body of the second-order forces. The second group of methods is based on the potential theory method, which, by using direct integration, calculates the fluid pressure on the floating body surface and the average of second-order wave forces, in which, these methods are nominated as near-field methods. There is also another method called the theory of potentials extracts the wave second-order forces by equating radiation damping energy and the work done by the wave collision. These methods are approximate and with the assumption of floating cylindrical body, the results will be appropriated. Finally, it is possible to propose approximate methods based on the Morrison equation and partial motion of second-order wave forces that are more suitable for lean semi-submersible structures. The force caused by wave radiation and diffraction has an important effect on floating objects in deep waters. The diffraction wave represents the dispersed term of a collision wave by a constant object. Besides, the radiation wave means that the wave generates through the fluctuation of a structure in stagnant water due to the collision of the incident wave. The forces caused by these phenomena can be determined by integrating the pressure on the surface of the floating structure using the diffraction and radiation potential function. Goodman presented the mean vertical force imposed to a semi-submersible cylinder due to collision of a lateral regular wave for wavelengths within the cylinder diameter range [2]. Dalzell et al. computed the components of the mean wave forces by Salvesen theory [3]. Comparison of numerical and experimental results shows the good accuracy of this theory. Pinkster extracted the relation between a low-frequency second-order wave drifting force and pressure due to the second order potential in the irregular wave [4]. Pijfers \& Brink, extracted relations based on the Morrison equation to calculate the second-order wave force on semi-submersible structures [5]. In an irregular sea condition that consists of regular wave components superposition, the high and low-frequencies second-order hydrodynamic forces, in the sum and the difference of frequencies formed from linear waves. These nonlinear waves can play an important role in stimulating some of the most important phenomena such as slow drift motion [6]. Several papers concern methods to investigate second-order interaction between waves and structure. Semi-analytical formulation for the second-order wave force applied to geometric fundamental structures by Taylor \& Kernot [7] using an indirect method [8], which is based on introduction of a radiation potential for calculating second-order wave forces and without explicit evaluation of second-order diffracted wave potential is presented.

Lim and Kim designed a new wave method for estimating the extreme slow-drift motion of moored offshore platforms [9]. The accuracy and applicability of this new method were tested through a series of simulations with a semi-submersible model. Jurado and Bredmose surveyed a numerical model that includes both invis- 
cid slow-drift forcing through full quadratic transfer functions (QTFs) and viscous forcing [10]. Zhang et al. studied low-frequency horizontal motions of a multi-point moored FPSO and the 2nd-order wave drift forces [11]. Renaud et al. developed mid-field formulas for multi-directional collision waves and carried out calculations for progressive wave forces and low frequency forces for a LNG carrier ship Lupton and Langley investigated the scaling of small drift motions with platform size with mooring characteristics and wave conditions [12]. Recently, Motallebi et al. calculated the second-order hydrodynamic force on fixed and floating tandem cylinders [13].

This paper presents the effect of variation in floating cylinder parameters (such as draft and radius). Low-frequency second-order hydrodynamic wave forces are applied on a floating cylinder. Figure 1 shows the floating cylinder under the incident wave effect.

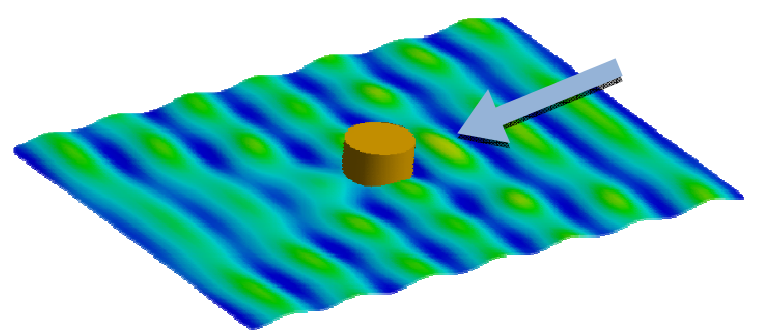

Fig. 1. Floating cylinder under incident wave effect

\section{Governing equations}

\subsection{Potential-based boundary element method}

Considering the Laplace equation as the governing equation, the velocity potential of the fluid flow field surrounds the floating object is defined as follows [14]:

$$
\Phi(\vec{X} t)=a_{w} \varphi(\vec{X}) e^{-i \omega t}
$$

where $a_{w}$ and $\omega$ are the incident wave amplitude and the wave frequency respectively. The expression $\varphi(\vec{X})$ is composed into three components include the first-order incident wave potential $\left(\varphi_{I}\right)$ with unit wave amplitude, the corresponding diffracted wave potential $\left(\varphi_{d}\right)$ and the radiation wave potential $\left(\varphi_{r j}\right)$ due to the $j$-th motion with unit motion amplitude. Therefore, the velocity potential equation may be written as:

$$
\varphi(\vec{X}) e^{-i \omega t}=\left[\varphi_{\mathrm{I}}+\varphi_{d}+\sum_{j=1}^{6} \varphi_{r j} x_{j}\right] e^{-i \omega t}
$$


By considering an incompressible, non-viscous and irrational flow, the velocity potential function is obtained from the Laplace equation solution in the whole fluid domain as follows [15]:

$$
\Delta \varphi=\frac{\partial^{2} \varphi}{\partial X^{2}}+\frac{\partial^{2} \varphi}{\partial Y^{2}}+\frac{\partial^{2} \varphi}{\partial Z^{2}}=0
$$

the fluid-structure interaction behavior is described by the following set of boundary conditions:

- $-\omega^{2} \varphi+\mathrm{g} \frac{\partial \varphi}{\partial Z}=0$ on $Z=0$

- $\frac{\partial \varphi}{\partial n}= \begin{cases}-i \omega n_{j} & \text { For radiation potential } \\ -\frac{\partial \varphi}{\partial n} & \text { For diffraction potential }\end{cases}$

- $\frac{\partial \varphi}{\partial Z}=0$ on $Z=-d$ (water depth)

- $|\nabla \varphi| \rightarrow 0$ when $\sqrt{x^{2}+y^{2}} \rightarrow \infty$

The boundary integration method is used in order to solve the fluid velocity potential function which is satisfied by boundary conditions. Applying Green's theorem, the velocity potential of wave radiation and diffraction can be represented as a Fredholm second kind integral equation:

$$
c \varphi(\vec{X})=\int_{S_{0}}\left\{\varphi(\vec{\xi}) \frac{\partial G(\vec{X} \cdot \vec{\xi} \cdot \omega)}{\partial n(\vec{\xi})}-G(\vec{X} \cdot \vec{\xi} \cdot \omega) \frac{\partial \varphi(\vec{\xi})}{\partial n(\vec{\xi})}\right\} d S
$$

where $G$ is Green's function. For more details, refer to Ghafari et al. [16].

\subsection{Drift forces}

The drift forces concept can be explained based on considering the hydrodynamic responses supposition of the fixed or floating body surrounding by an incompressible, irrotational, homogeneous and inviscid fluid. Furthermore, in this hypothesis, both body motion responses amplitude and the fluid wave amplitude are small. With these assumptions, the velocity potential function can be used to express the surrounding fluid. For the sake of declaring the wave elevation, the position of a point on a hull body and the fluid potential, the perturbation approach is applied as below:

$$
\begin{aligned}
& \Phi=\varepsilon \Phi^{(1)}+\varepsilon^{2} \Phi^{(2)}+O\left(\varepsilon^{3}\right) \\
& \zeta=\zeta^{(0)}+\varepsilon \zeta^{(1)}+\varepsilon^{2} \zeta^{(2)}+O\left(\varepsilon^{3}\right) \quad \text { where } \varepsilon \rightarrow 0 \\
& \vec{X}=\vec{X}^{(0)}+\varepsilon \vec{X}^{(1)}+\varepsilon^{2} \vec{X}^{(2)}+O\left(\varepsilon^{3}\right)
\end{aligned}
$$


where $\varepsilon$ is the perturbation parameter and the static values, the first and the second order variations with respect to $\varepsilon$, denoting by superscripts (0), (1) and (2), respectively.

$$
\vec{X}^{(0)}=\vec{X}_{g}^{(0)}+\vec{x} \quad \vec{X}^{(1)}=\vec{X}_{g}^{(1)}+\vec{\alpha}^{(1)} \times \vec{x}
$$

where, $\vec{X}_{g}^{(0)}, \vec{X}_{g}^{(1)}$ and $\vec{\alpha}^{(1)}$ are the static position, the first order body center of gravity translational and rotational motion in fixed reference axis, respectively. The point position can be defined by $\vec{x}$ in local structure axes. It should be noted that $\vec{X}^{(2)}$ has similar forms as those in Eq. (7).

The first-order velocity $\dot{\vec{X}}^{(1)}$, response acceleration, $\ddot{\vec{X}}^{(1)}$ and the first-order component of the normal vector, $\vec{N}^{(1)}$ in fixed reference axes in a location of the hull surface are written as follows

$$
\dot{\vec{X}}^{(1)}=\dot{\vec{X}}_{g}^{(1)}+\dot{\vec{\alpha}}^{(1)} \times \vec{x}, \quad \ddot{\vec{X}}^{(1)}=\ddot{\vec{X}}_{g}^{(1)}+\ddot{\vec{\alpha}}^{(1)} \times \vec{x}, \quad \vec{N}^{(1)}=\vec{\alpha}^{(1)} \times \vec{n}
$$

The fluid pressure at a specific point is measured on the basis of Bernoulli's equation, which can be represented by the Taylor series in Eq. (9):

$$
p=-\rho \frac{\partial \Phi}{\partial t}-\frac{1}{2} \rho \nabla \Phi . \nabla \Phi-\rho g Z=\mathrm{p}^{(0)}+\mathrm{p}^{(1)}+\mathrm{p}^{(2)}+\mathrm{O}\left(\varepsilon^{3}\right)
$$

Since:

$$
\begin{aligned}
& \mathrm{p}^{(0)}=-\rho g X_{3}^{(0)} \\
& \mathrm{p}^{(1)}=-\rho g X_{3}^{(1)}-\rho \frac{\partial \Phi^{(1)}}{\partial t} \\
& \mathrm{p}^{(2)}=-\frac{1}{2} \rho\left|\nabla \Phi^{(1)}\right|^{2}-\rho \vec{X}^{(1)} \cdot \nabla \frac{\partial \Phi^{(1)}}{\partial t}-\rho \frac{\partial \Phi^{(2)}}{\partial t}-\rho g X_{3}^{(2)}
\end{aligned}
$$

Let's define $s(t)$ and $\vec{N}$ as the instantaneous wetted surface and unit normal vector, respectively. The total fluid force and moment in the fixed reference axes can be expressed as follows:

$$
\begin{gathered}
\bar{F}(t)=-\iint_{S(t)} p \vec{N} d S \\
\bar{M}(t)=-\iint_{S(t)} p\left[\left(\vec{X}-\vec{X}_{g}\right) \times \vec{N}\right] d S
\end{gathered}
$$

By using the perturbation series analysis of Eqs. (11) and (12), over the wetted surface $s(t)$, the second-order wave exciting force and moment can be calculated in the following manner [1]: 


$$
\begin{aligned}
& \vec{F}^{(2)}=-\frac{1}{2} \rho g \oint_{W L} \zeta_{r}^{(1)} \cdot \zeta_{r}^{(1)} \vec{n} d l \quad \text { Water line integral } \\
& +\frac{1}{2} \rho \iint_{S_{0}}\left[\nabla \Phi^{(1)} \cdot \nabla \Phi^{(1)}\right] \vec{n} d S \quad \text { Bernoulli } \\
& +\rho \iint_{S_{0}}\left[\vec{X}^{(1)} \cdot \nabla \frac{\partial \Phi^{(1)}}{\partial t}\right] \vec{n} d S \quad \text { Acceleration } \\
& +\vec{\alpha}^{(1)} \times \vec{F}^{(1)} \quad \text { Momentum } \\
& +\rho \iint_{S_{0}} \frac{\partial \Phi^{(2)}}{\partial t} \vec{n} d S \quad \text { Second-order potential } \\
& \vec{M}^{(2)}=-\frac{1}{2} \rho g \oint_{W L} \zeta_{r}^{(1)} \cdot \zeta_{r}^{(1)}(\vec{x} \times \vec{n}) d l \quad \text { Water line integral } \\
& +\frac{1}{2} \rho \iint_{S_{0}}\left[\nabla \Phi^{(1)} \cdot \nabla \Phi^{(1)}\right](\vec{x} \times \vec{n}) d S \quad \text { Bernoulli } \\
& +\rho \iint_{S_{0}}\left[\vec{X}^{(1)} \cdot \nabla \frac{\partial \Phi^{(1)}}{\partial t}\right](\vec{x} \times \vec{n}) d S \quad \text { Acceleration } \\
& +\vec{\alpha}^{(1)} \times \vec{M}^{(1)} \quad \text { Momentum } \\
& +\rho \iint_{S_{0}} \frac{\partial \Phi^{(2)}}{\partial t}(\vec{x} \times \vec{n}) d S \quad \text { Second-order potential }
\end{aligned}
$$

where $S_{0}$ is the mean wetted surface, $\zeta_{r}^{(1)}=\zeta^{(1)}-X_{3 W L}^{(1)}$ is the relative wave elevation along the mean undisturbed water line, $\vec{F}^{(1)}$ and $\vec{M}^{(1)}$ are the total first order fluid force and moment, respectively.

\subsection{Equations of motion}

Through the interaction of the body motion within frequency dependent hydrodynamic coefficients, a set of linear motion equations can be derived as follows:

$$
\left[-\omega_{e}^{2}\left(M_{s}+M_{a}\right)-i \omega_{e} C+K_{h y s}\right]\left[x_{j}\right]=\left[F_{j}\right]
$$

In Eq. (15), $M_{s}$ is a $6 \times 6$ body mass matrix, $M_{a}=\left[A_{j, k}\right]$ and $C=\left[B_{j, k}\right]$ are the $6 \times 6$ hydrodynamic added mass and damping matrices, $K_{h y s}$ is hydrostatic stiffness matrix. $F_{j}$ is the total forces and moments. $\omega_{e}$ is the encountering frequency, and the encountering period is $T_{e}=\frac{2 \pi}{\omega_{e}}$. Here, in our case, the wave period and encountering period are the same $\left(T_{e}=T\right)$. 


\section{Numerical results}

\subsection{Validation}

In this paper for verification purposes, the experimental test results of the restrained cylinder body are used as representative of a vertical cylinder [17]. The experimental work for the restrained body carried out at the offshore water wave basin of the Danish Hydraulic Institute (DHI). This basin is $3 \mathrm{~m}$ in depth, $30 \mathrm{~m}$ in length and $20 \mathrm{~m}$ in width and includes a hydraulic flap wave maker. As shown in Figure 3, the model was placed in the basin by using the triangular shaped rigid rig.

a)

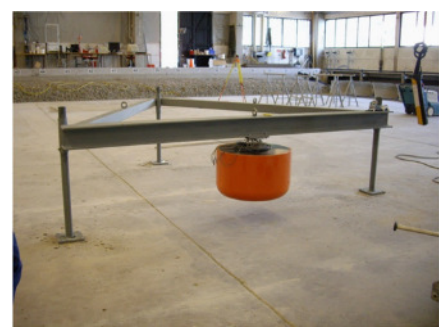

b)

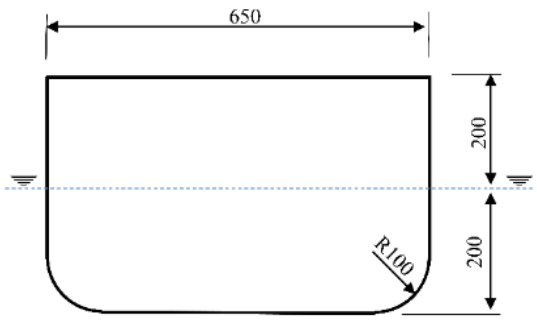

Fig. 3. a) Photo of tested body [17]. b) Schematic model (dimensions in mm)

The applied mean drift forces in the heave and surge directions, due to radiation of the monochromatic wave, were measured. Table 1 shows the range of the incident wave periods according to [17].

Table 1. Characteristics of the monochromatic tested waves

\begin{tabular}{|c|c|c|}
\hline Wave index & Period $[\mathrm{s}]$ & Amplitude $[\mathrm{cm}]$ \\
\hline 1 & 0.70 & 1.1 \\
\hline 2 & 0.90 & 1.6 \\
\hline 3 & 1.10 & 2.2 \\
\hline 4 & 1.15 & 2.3 \\
\hline 5 & 1.35 & 2.9 \\
\hline 6 & 1.75 & 4.0 \\
\hline 7 & 1.90 & 4.4 \\
\hline 8 & 2.00 & 4.7 \\
\hline 9 & 2.20 & 4.8 \\
\hline 10 & 1.55 & 3.4 \\
\hline
\end{tabular}

Figure 4 shows the comparison of the non-dimensional drift force coefficients $\left(F_{2 n d}\right)$, heave and surge drift forces as function of the wave period. The forces are non-dimensionalized as: $\frac{F_{2 n d}}{\rho g A^{2} L}$, where $g, \rho$ and $A$ are gravity acceleration, fluid density and wave amplitude, respectively. $L$ is defined as characteristic length and 
is considered equal to $1 \mathrm{~m}$. Figure 4 shows the comparison of the numerical and experimental drift force of the restrained cylinder body in both surge and heave directions. The results show that there is good agreement between the numerical results and experimental data.
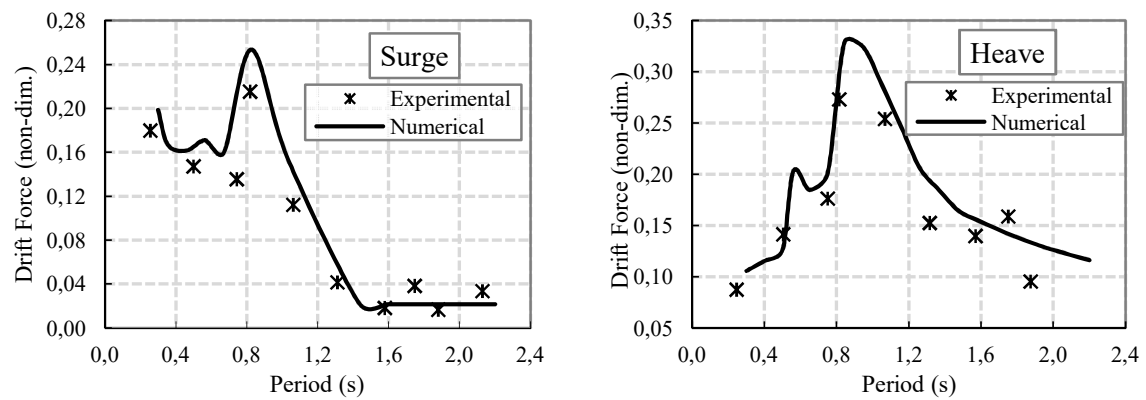

Fig. 4. Comparison of non-dimensional drift force for surge and heave motions

\subsection{Effect of draft and radius}

The applied drift force on the floating cylinder body has been investigated for eight different cylinder drafts (with the constant radius of $1 \mathrm{~m}$ ) and five different radiuses (with the constant draft of $2 \mathrm{~m}$ ) in frequency-domain analysis. The intended cylinder has 6 degrees of freedom. The results of non-dimensional applied forces for different drafts and radiuses are shown in Figures 5 and 6, respectively.
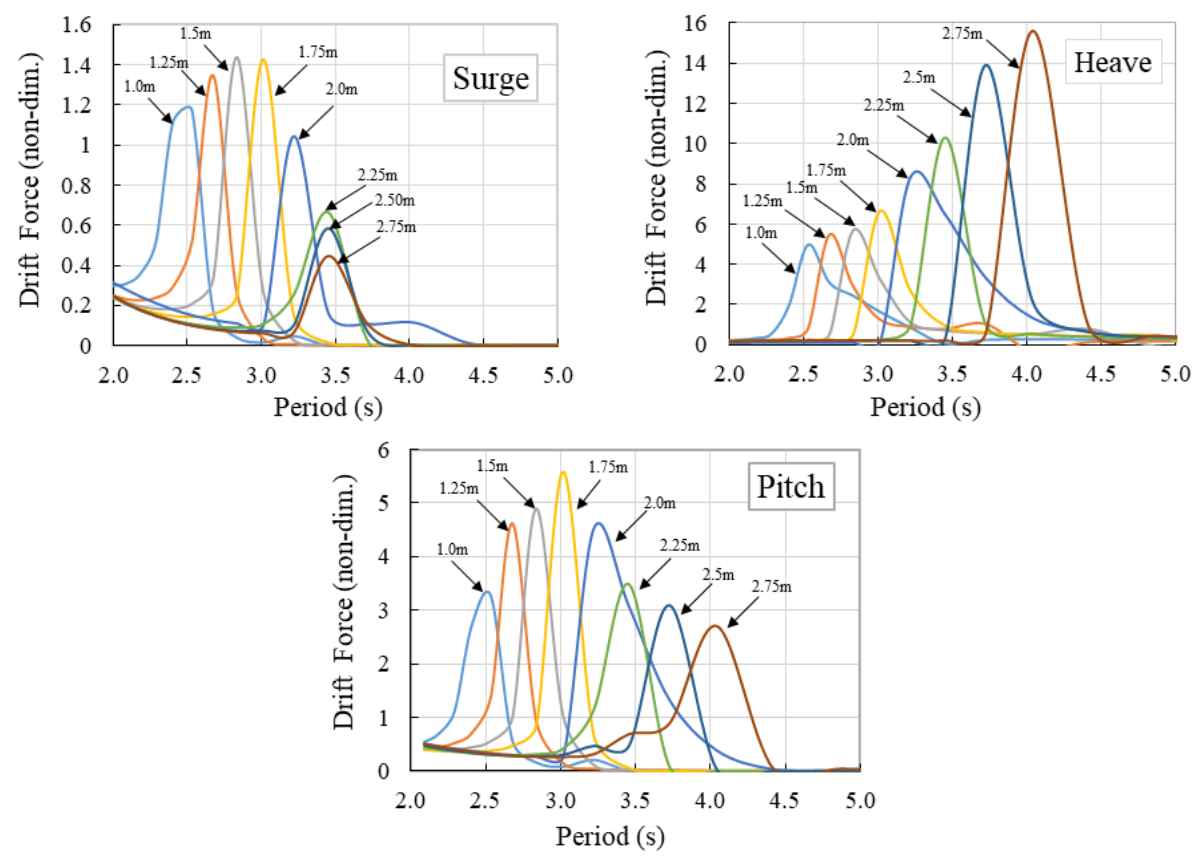

Fig. 5. Drift forces on a floating cylinder versus different drafts (constant radius) 
As shown in Figure 5, by increasing the draft in the constant radius, the amount of drift force on a floating cylinder in surge direction at first increased and reached a maximum value of 1.43 in both $1.5 \mathrm{~m}$ and $1.75 \mathrm{~m}$ draft cylinders, then the amount of exerted force gradually declines by almost $70 \%$ in a $2.75 \mathrm{~m}$ draft.
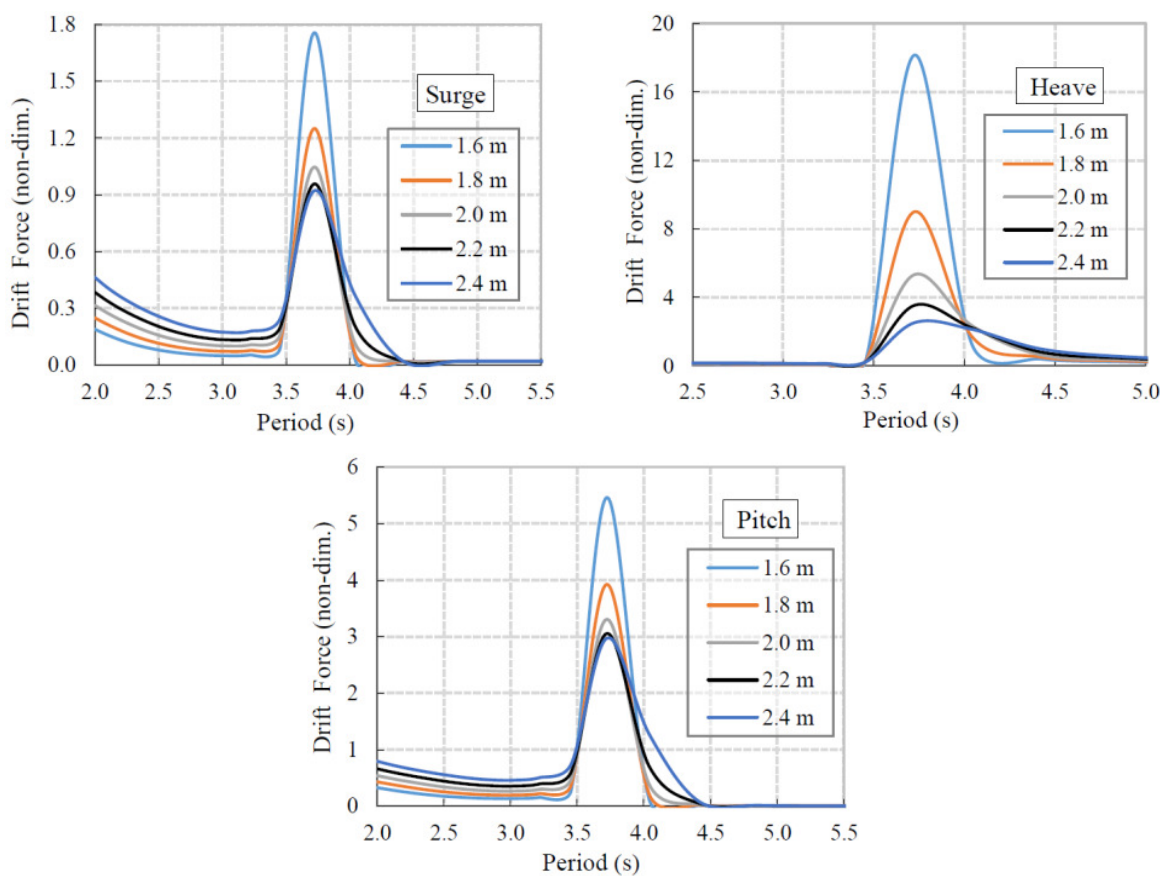

Fig. 6. Drift forces on a floating cylinder versus different radiuses (constant draft)

According to Figure 6 with rising radiuses of cylinders in the constant draft, the values of the drift force are decreased in all three directions of the surge, heave and pitch, while the period of maximum force remained constant at about $3.75 \mathrm{~s}$. It can be seen that by increasing the radius from $1.6 \mathrm{~m}$ to $2.4 \mathrm{~m}$, the drift force is reduced by nearly $45 \%$ in both surge and pitch directions, while in heave direction, $85 \%$ reduction is visible. The results are summarized in Figure 7.
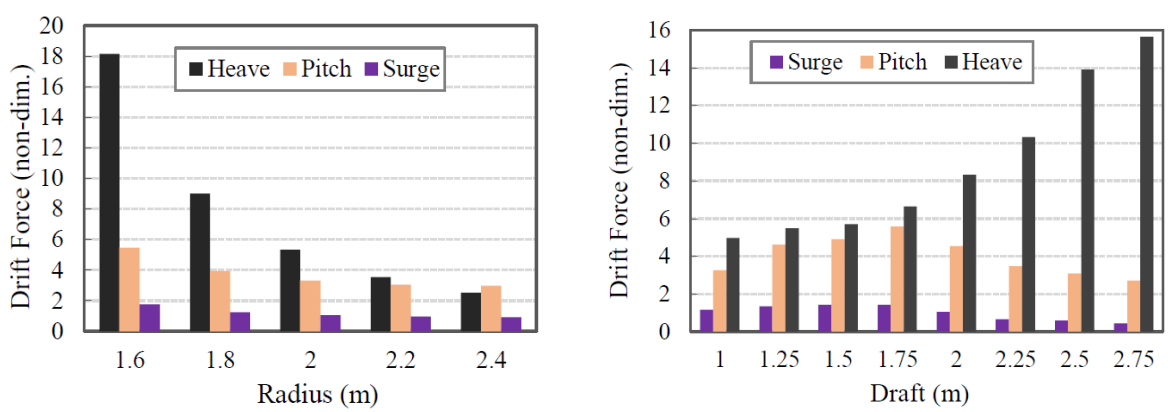

Fig. 7. Maximum drift forces on a floating cylinder versus different radiuses and drafts 


\section{Conclusion}

This paper is presented to calculate the drift forces of the single floating cylinder with different drafts and radiuses. The following conclusions can be drawn:

- The numerical results show good agreement with experimental data for drift force of surge and heave motions.

- The maximum drift force occurrs at the period $3.75 \mathrm{~s}$ for all drafts of a cylinder. The lower drafts make more force.

- The effect of the radius is different. By increasing the radius, the maximum drift forces are obtained at different periods.

\section{References}

[1] Pinkster, J.A. (1980). Low-frequency second order wave exciting forces on floating structures. $\mathrm{PhD}$ thesis, MARIN Publication No. 600.

[2] Goodman, T.R. (1962). Forces on a Hovering Slender Body of Revolution Submerged Under Waves of Moderate Wavelength (No. 63). Ocean Research Equipment Inc Vineyard Haven Ma.

[3] Dalzell, J.F., \& Kim, C.H. (1976). Analytical Investigation of the Quadratic Frequency Response for Added Resistance (No. SIT-DL-76-1878). Stevens Institute of Tech Hoboken NJ Davidson Lab.

[4] Pinkster, J.A. (1976). Low frequency second order wave forces on vessels moored at sea. Proc. of the $11^{\text {th }}$ Symp. on Naval Hydrodynamics.

[5] Pijfers, J.G., \& Brink, A.W. (1977). Calculated drift forces of two semisubmersible platform types in regular and irregular waves. In Offshore technology conference. Offshore Technology Conference.

[6] Petrauskas, C., \& Liu, S.V. (1987). Springing force response of a tension leg platform. In Offshore Technology Conference. Offshore Technology Conference.

[7] Taylor, R.E., \& Kernot, M.P. (1999). On second order wave loading and response in irregular seas. In Advances in Coastal and Ocean Engineering (pp. 155-212).

[8] Molin, B. (1979). Second-order diffraction loads upon three-dimensional bodies. Applied Ocean Research, 1(4), 197-202.

[9] Lim, D. H., \& Kim, Y. (2018). Design wave method for the extreme horizontal slow-drift motion of moored floating platforms. Applied Ocean Research, 71, 48-58.

[10] Pegalajar-Jurado, A., \& Bredmose, H. (2019). Reproduction of slow-drift motions of a floating wind turbine using second-order hydrodynamics and operational modal analysis. Marine Structures, 66, 178-196.

[11] Zhang, L., Lu, H., Yang, J., Peng, T., \& Xiao, L. (2013). Low-frequency drift forces and horizontal motions of a moored FPSO in bi-directional swell and wind-sea offshore West Africa. Ships and Offshore Structures, 8(5), 425-440.

[12] Renaud, M., Waals, O., Chen, X.B., \& van Dijk, R. (2008). Second-order wave loads on a lng carrier in multi-directional waves. In ASME 2008 27th International Conference on Offshore Mechanics and Arctic Engineering (pp. 363-370). American Society of Mechanical Engineers Digital Collection.

[13] Motallebi, M., Ghafari, H.R., Ghassemi, H., \& Shokouhian, M. (2020). Calculating the secondorder hydrodynamic force on fixed and floating tandem cylinders. Scientific Journals of the Maritime University of Szczecin, 62(134), 108-115. 
[14] Barltrop, N.D. (Ed.). (1998). Floating structures: a guide for design and analysis (Vol. 1). Oilfield Publications Incorporated.

[15] Newman, J.N. (1979). The theory of ship motions. In Advances in Applied Mechanics (Vol. 18, pp. 221-283). Elsevier.

[16] Ghafari, H.R., Ketabdari, M.J., Ghassemi, H., \& Homayoun, E. (2019). Numerical study on the hydrodynamic interaction between two floating platforms in Caspian Sea environmental conditions. Ocean Engineering, 188(106273).

[17] Fonseca, N., Pessoa, J., Mavrakos, S., \& Le Boulluec, M. (2011). Experimental and numerical investigation of the slowly varying wave exciting drift forces on a restrained body in bi-chromatic waves. Ocean Engineering, 38(17-18), 2000-2014. 